\title{
Tomato genomic prediction for good performance under high-temperature and identification of loci involved in thermotolerance response
}

Elisa Cappetta ${ }^{1,2}$, Giuseppe Andolfo ${ }^{1}$, Anna Guadagno ${ }^{1}$, Antonio Di Matteo $\mathbb{1}^{1}{ }^{1}$, Amalia Barone ${ }^{1}$, Luigi Frusciante ${ }^{1}$ and Maria Raffaella Ercolano (1) ${ }^{1 凶}$

\begin{abstract}
Many studies showed that few degrees above tomato optimum growth temperature threshold can lead to serious loss in production. Therefore, the development of innovative strategies to obtain tomato cultivars with improved yield under high temperature conditions is a main goal both for basic genetic studies and breeding activities. In this paper, a F4 segregating population was phenotypically evaluated for quantitative and qualitative traits under heat stress conditions. Moreover, a genotyping by sequencing (GBS) approach has been employed for building up genomic selection (GS) models both for yield and soluble solid content (SCC). Several parameters, including training population size, composition and marker quality were tested to predict genotype performance under heat stress conditions. A good prediction accuracy for the two analyzed traits ( 0.729 for yield production and 0.715 for SCC) was obtained. The predicted models improved the genetic gain of selection in the next breeding cycles, suggesting that GS approach is a promising strategy to accelerate breeding for heat tolerance in tomato. Finally, the annotation of SNPs located in gene body regions combined with QTL analysis allowed the identification of five candidates putatively involved in high temperatures response, and the building up of a GS model based on calibrated panel of SNP markers.
\end{abstract}

\section{Introduction}

Tomato (Solanum lycopersicum) is one of the most important worldwide horticulture crops ${ }^{1}$. However, this crop face with high production losses caused by heat stress $^{2,3}$, which heavily impact tomato fruit setting and yield related traits ${ }^{4,5}$. Indeed, temperature exceeding $30^{\circ} \mathrm{C}$ during the day and $21^{\circ} \mathrm{C}$ during the night can be very challenging for crop production ${ }^{6}$. Hence, heat stress is considered as a bottleneck factor for tomato fruit production, especially in temperate regions. Nowadays, developing tomato cultivars with improved yield under high temperature conditions may be a valuable strategy to cope with climate changes. The traditional plant breeding

\footnotetext{
Correspondence: Maria Raffaella Ercolano (ercolano@unina.it)

${ }^{1}$ Department of Agricultural Sciences, University of Naples Federico II, Via Università 100, 80055 Portici, Naples, Italy.

${ }^{2}$ Present address: Institute of Bioscience and BioResources, National Research

Council, Via Università 100, 80055 Portici, Italy
}

method for heat tolerance is based on the selection of advanced breeding lines showing greater yield performances than the current cultivars in a hot target production area. The plant evaluation is based on assessment of different quantitative and qualitative parameters such as pollen viability, fruit set, yield productivity per plant, total number of fruits, fruit shape, and soluble solid content ${ }^{7}$.

High-temperature tolerance is controlled by multiple genes which induce several physiological and biochemical changes, making the heat stress response hard to investigate $^{8}$. For these reasons, conventional breeding approaches resulted inadequate to meet the growing demand for heat tolerant (HT) varieties, since time-consuming phenotypic evaluation rounds and complex selection schemes are needed $^{9}$. In this regard, understanding the molecular mechanisms regulating the relationships among agronomic

\section{(c) The Author(s) 2021}

(c) (i) Open Access This article is licensed under a Creative Commons Attribution 4.0 International License, which permits use, sharing, adaptation, distribution and reproduction cc) in any medium or format, as long as you give appropriate credit to the original author(s) and the source, provide a link to the Creative Commons license, and indicate if changes were made. The images or other third party material in this article are included in the article's Creative Commons license, unless indicated otherwise in a credit line to the material. If material is not included in the article's Creative Commons license and your intended use is not permitted by statutory regulation or exceeds the permitted use, you will need to obtain permission directly from the copyright holder. To view a copy of this license, visit http://creativecommons.org/licenses/by/4.0/. 
important traits can rapidly increase the selection of new genotypes.

In the past 10 years, significant progress has been made in precision breeding and in selection methods for early release of crop varieties ${ }^{10}$. Innovative breeding strategies such as marker-assisted selection (MAS), highthroughput genotyping and phenotyping platforms, reverse breeding, and genomic selection, are increasingly being used to complement the conventional approaches to bring the release of crop varieties to an important step forward $^{11-14}$.

Tomato is a relative short life cycle crop and a high selffertility species with a well know genome structure, offering the possibility to design more tailored crop improvement approach ${ }^{15}$. Several QTLs for abiotic environmental stresses (such as salinity, drought, and heat) and for fruit-related characteristics have been reported in this species ${ }^{16-20}$. However, QTLs related to complex traits are hard to introgress in cultivated crops, also by using MAS. This is especially due to the large number of small effect genes controlling heat tolerance $^{21,22}$. Genomic selection (GS) is providing new opportunities for increasing the efficiency of plant breeding programs by overcome limiting factors ${ }^{23-26}$. The approach is based on estimating breeding values of an individual using genomics and phenotypic information. A training population (TRN) is evaluated at genotypic and phenotypic level to obtain a training set (TRS) and a test set (TST) used only for validation (i.e., evaluation of the accuracy). The trained GS model will be then applied to predict breeding values of individuals in the next selection step $^{27}$. The basic idea of a GS approach is to use genomewide marker data to predict genetic architecture of the TRN to effectively select the best individuals with superior traits. Thus, GS results in a robust approach to enhance the rate of genetic gain per unit of time and reduce the time required for screening superior individuals in breeding programs ${ }^{14}$. Successfully examples of GS approaches have been reported in several crops ${ }^{26,28-30}$, including tomato ${ }^{31-34}$. In addition, empirical studies have also demonstrated that GS has higher genetic gain than MAS for complex traits controlled by large number of QTLs $^{35}$. However, although genotyping cost have decreased significantly in recent years, it is still a very onerous phase to carry out that slow down the overall GSbased breeding process. In theory, the GS prediction accuracy improves with higher genetic markers density, but several empirical studies showed that in population with long haplotypes overlap among individuals, accuracy seems to be relatively stable when a well calibrate set of markers is chosen ${ }^{36-41}$.

At light of these observations and to better investigate the basis of tomato heat stress tolerance, a F4 segregating population was phenotypically evaluated for quantitative and qualitative traits under heat stress condition. Then, a genotyping by sequencing (GBS) approach has been employed to produce de novo molecular markers useful to build up a suitable GS models for accelerating the selection of heat tolerant individuals. Several critical parameters such as size of training population, number and quality of markers were carefully evaluated. Subsequently, trained models were applied to the next generations to identify superior genotypes and a calibrated SNP subset was selected and tested to build up a model prediction. Finally, the annotation of SNPs located in gene regions was integrated with a QTL analyses to identify genes putatively involved in heat stress tolerance.

\section{Results}

Analysis of phenotypic traits in tomato segregating population for heat stress tolerance

In this study, a F4 tomato population deriving from single seed selection schema performed on the heat tolerant tomato variety JAG8810, was grown under high temperatures. Tomato plants were transplanted in June in the open fields under plastic tunnel with a delay of one month compared to the usual transplanting period (April) of the area to impose spontaneously occurring heat stressing conditions. As reported in Supplementary Fig. 1, the temperature was mostly upper than $30^{\circ} \mathrm{C}$ during the day and exceeded the critical threshold level of $35^{\circ} \mathrm{C}$ in 11 out of 81 days. Under these conditions, the JAGF4 lines were phenotyped for 8 traits, mainly related to fruit production and fruit quality. A wide range of variability in the eight production-related traits was observed and reported in Table 1 and Table S1.

Distributions without any significant skewness were observed for all analyzed traits except for inflorescence number and fruit earliness (Fig. 1).

Interestingly, significant correlations among parameters related to fruit production were found (Fig. 2). In particular, Pearson's correlation coefficient $(r)$ ranged from the lowest correlation $(r=-0.53)$ between yield production per plant (YP) and contemporaneous ripening (CR) to the highest

\begin{tabular}{llll}
$\begin{array}{l}\text { Table } \mathbf{1} \\
\text { related to fruit set, total fruit number per plant, yield per } \\
\text { plant, and soluble solid content }\end{array}$ & & Min & Max \\
\hline Trait & Mean & Minimum and maximum values of traits \\
\hline FS (\%) & 38.9 & 8 & 78 \\
TFN & 158.2 & 32 & 354 \\
YP (Kg/plant) & 8.0 & 0.8 & 16 \\
SSC & 4.4 & 3 & 6.8 \\
\hline
\end{tabular}

FS (\%) fruit set percentage, TFN total fruit number per plant, YP yield production per plant, SSC soluble solid content 

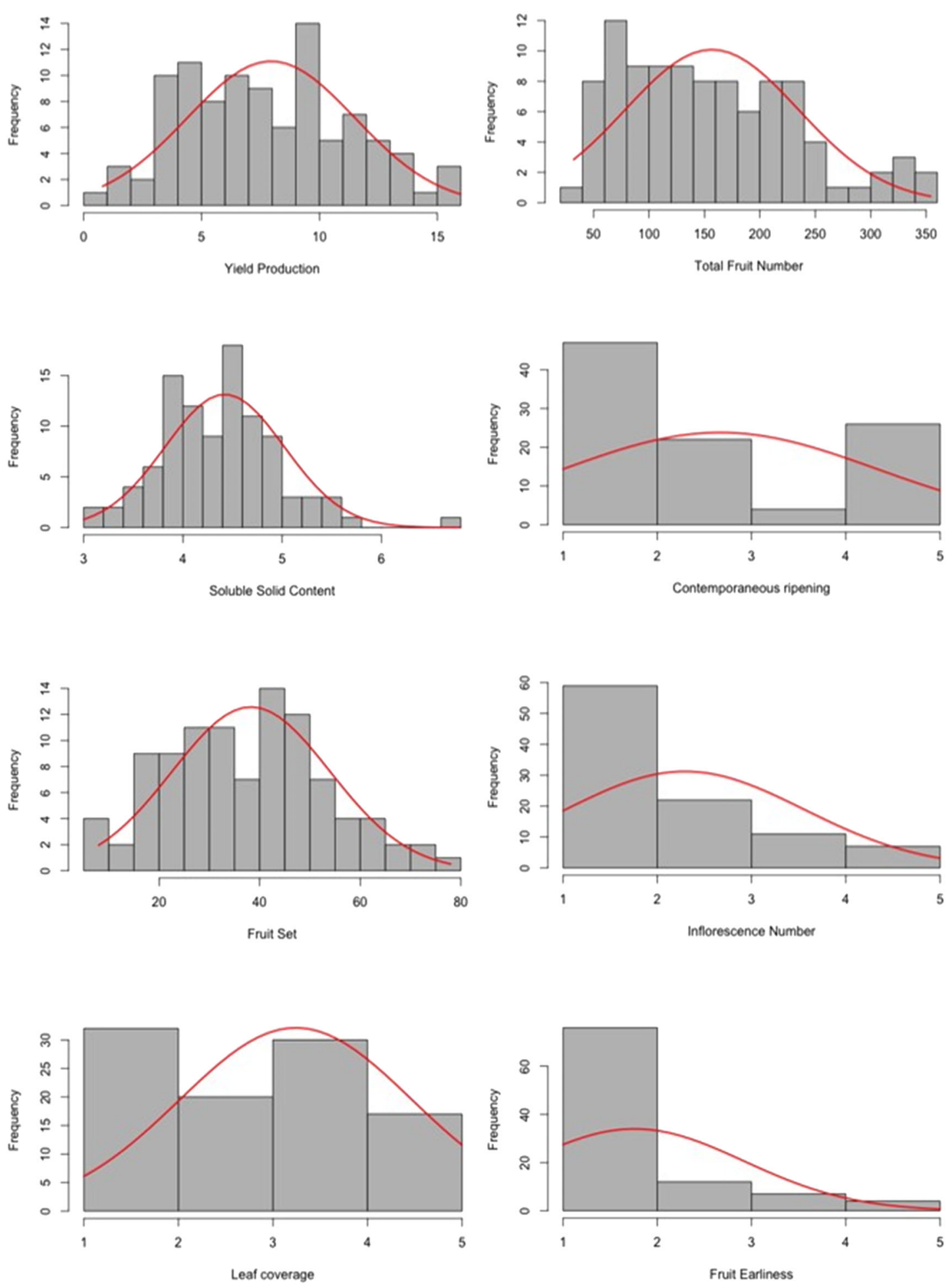

Fig. 1 Frequency distribution of the phenotypic value of eight traits of interest. X-axis show phenotypic values whereas in the $Y$-axis the frequency is reported

correlation $(r=0.89)$ between YP and total fruit number per plant (TFN) (Table S2). YP also significantly correlated with percentage of fruit set (FS) $(r=0.58)$, inflorescence number
(IN) $(r=0.38)$ and fruit earliness (FRL) $(r=0.33)$ but negative correlated with soluble solid content (SSC) $(r=-0.33)$. SSC negatively correlated with other yield-related traits such 


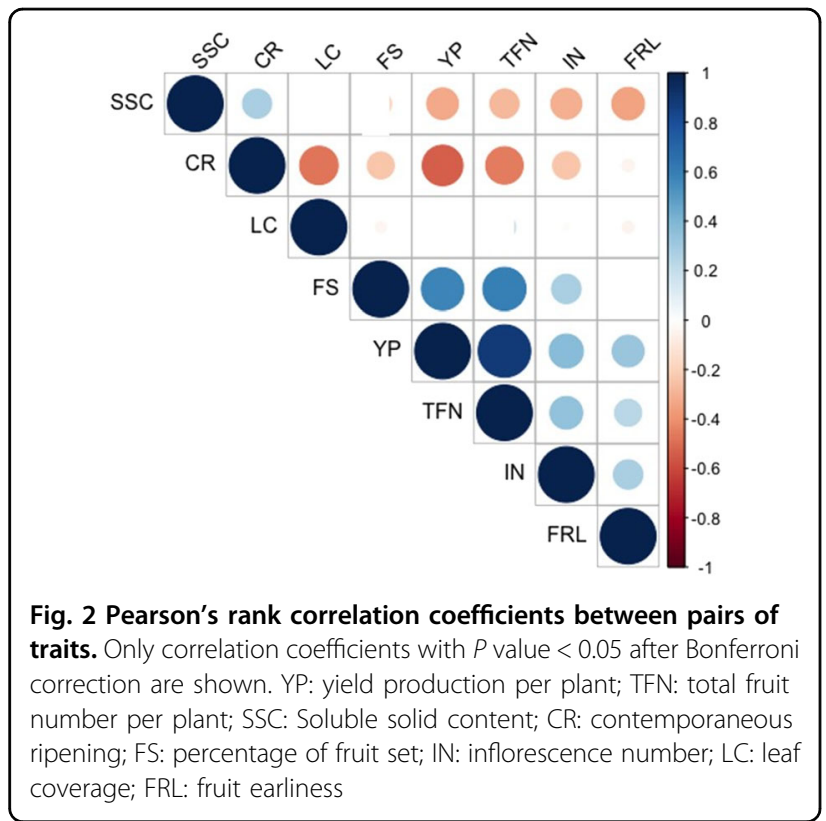

as TFN $(r=-0.28)$, IN $(r=-0.30)$ and FRL $(r=-0.35)$. However, we found a weak positive correlation between SSC and CR $(r=0.27)$, which instead negative correlated with leaf coverage (LC) $(r=-0.46)$.

\section{High-throughput genotyping of JAGF4 and JAGF5 populations}

The hybrid variety JAG8810, 100 JAGF4, and 54 JAGF5 single individuals were genotyped by GBS approach producing a total of $117,798,988$ and $136,926,433$ reads in JAGF4 and JAGF5 populations respectively. After quality check, all reads were mapped onto the reference genome (Solanum lycopersicum cv. Heinz 1706 v3.0), and the results were used for SNP and INDELS discovery. Overall, 135,255 SNPs in JAGF4 and 176,596 SNPs in JAGF5 populations were found (Table 2).

Their distribution along chromosomes is displayed in Fig. 3 showing a similar SNP density between two populations except for chromosome 9 where a higher SNP density was evident in JAGF5 compared with JAGF4 population. SNP number per chromosome ranged from 2519 (chromosome 9) to 22,500 (chromosome 6) with an average of 7278 in JAGF4 population. Instead, SNP number per chromosome varied from 3956 (chromosome 10) to 27,373 (chromosome 6) with an average of 10,736 in JAGF5 (Table 2). In JAGF4 the minimum average polymorphism information content (PIC) and heterozygous values $(\mathrm{H})$ were encountered on chromosome 9 ( 0.136 and 0.171 , respectively), while the maximum values were found on chromosome 5 ( 0.313 and 0.410 , respectively). Similarly, in JAGF5 the minimum values were encountered on chromosome $9 \quad(0.022$ and 0.028 respectively) whereas the maximum PIC and $\mathrm{H}$ values were found on chromosome 5 (0.28 and 0.369, respectively).

The heterozygous/homozygous number ratio and the SNP number per sample were also displayed in Fig. 4a and Fig. $4 \mathrm{~b}$ showing similar trends in the two populations. Also, the observed transitions/transversions (Ts/Tv) ratios in two studied populations were similar (Fig. 4c). In particular, we found a transitions/transversions (Ts/Tv) ratio of 1.19 in JAGF4 and 1.21 in JAGF5 with a total of 73,392 transitions and 61,863 transversions events in JAGF4 and 96,710 transitions and 79,886 transversions events in JAGF5 (Table S3). Among transitions events, $\mathrm{C}>\mathrm{T}$ and $\mathrm{G}>\mathrm{A}$ were the most abundant, whereas $\mathrm{C}>\mathrm{A}$ and $\mathrm{G}>\mathrm{T}$ were the most frequently transversion events in both populations (Fig. 4d). After removing, SNPs with minor allele frequency (MAF) less than 5\%, a total of 101,797 and 109,967 SNPs in JAGF4 and JAGF5 population, respectively, were obtained.

\section{GS model training using the JAGF4 population}

The JAGF4 genotypic and phenotypic data were used to train a prediction model for identifying the most suitable candidates for next-generation selection round (GS train). Given the positive correlations observed among the yieldrelated traits and the negative correlation between yield and the soluble solid content (SSC), we investigated the optimal design of GS models for YP and SSC traits using a TRN population of 90 and 100 individuals, respectively. As for the training of the GS models, the interaction between different sets of TRN composition and marker dataset were examined using a $5 \times 5$ contingency table. First, SNP dataset was filtered for five levels of percentage of eliminated missing values (PEMV) $(70,75,80,85,90 \%)$ obtaining five different marker datasets for each trait (Table S4). Five TRN subsets of different composition were then obtained for each trait varying the TRS $\backslash T S T$ ratio. TRS datasets ranging from 60 to 80 and 55 to 75 sampled genotypes for SSC and YP, respectively, were obtained randomly removing 5 genotypes to each round.

In this way, twenty-five datasets using 5 TRS $\backslash T S T$ subsets of different composition and 5 PEMV filtered marker sets were assessed for each trait. To evaluate the prediction ability of RR-blup models to estimate the genetic breeding values (GEBVs), 1000 iteration cycles were run. The results revealed that predictive ability did steadily increase up until the maximum PEMV and larger TRS in the hold-out validation strategy for both traits. Indeed, prediction accuracy was maximum for the following combinations: PEMV $90 \%$ with a SNPs dataset of 14,286 and TRS $\backslash$ TST composition in $80 \backslash 20$ ratio for SSC and PEMV 90\% with a SNP dataset of 14,210 and TRS ITST composition in $75 \backslash 15$ ratio for YP (Fig. 5). Genomic prediction accuracy in our trained models, defined here as 
Table 2 SNP distribution along tomato chromosomes in JAGF4 and JAGF5 populations

\begin{tabular}{|c|c|c|c|c|c|c|c|c|c|}
\hline Chromosome & Length (bp) & $\begin{array}{l}\text { SNP } \\
\text { number JAGF4 }\end{array}$ & $\begin{array}{l}\text { SNP } \\
\text { number JAGF5 }\end{array}$ & $\%$ SNP JAGF4 & $\%$ SNP JAGF5 & H JAGF4 & PIC JAGF4 & H JAGF5 & PIC JAGF5 \\
\hline Chro & 20852292 & 47913 & 47753 & 35.42 & 27.04 & 0.316 & 0.249 & 0.292 & 0.229 \\
\hline Chr1 & 98455869 & 5794 & 7238 & 4.28 & 4.1 & 0.252 & 0.199 & 0.21 & 0.166 \\
\hline Chr2 & 5597758 & 4119 & 4922 & 3.04 & 2.79 & 0.264 & 0.206 & 0.223 & 0.174 \\
\hline Chr3 & 72290146 & 4596 & 5882 & 3.4 & 3.33 & 0.269 & 0.211 & 0.202 & 0.158 \\
\hline Chr4 & 66557038 & 9892 & 12359 & 7.31 & 7 & 0.396 & 0.302 & 0.361 & 0.276 \\
\hline Chr5 & 66723567 & 13763 & 17481 & 10.18 & 9.9 & 0.410 & 0.313 & 0.369 & 0.28 \\
\hline Chr6 & 49794276 & 22500 & 27373 & 16.64 & 15.5 & 0.343 & 0.269 & 0.332 & 0.258 \\
\hline Chr7 & 68175699 & 2692 & 5142 & 2 & 2.91 & 0.188 & 0.149 & 0.113 & 0.09 \\
\hline Chr8 & 65987440 & 3676 & 4533 & 2.72 & 2.57 & 0.236 & 0.186 & 0.214 & 0.168 \\
\hline Chr9 & 72906345 & 2519 & 19947 & 1.86 & 11.29 & 0.171 & 0.136 & 0.028 & 0.022 \\
\hline Chr10 & 65633393 & 2776 & 3956 & 2.05 & 2.24 & 0.193 & 0.153 & 0.158 & 0.126 \\
\hline Chr11 & 56597135 & 10946 & 13278 & 8.09 & 7.52 & 0.339 & 0.262 & 0.305 & 0.238 \\
\hline Chr12 & 68126176 & 4069 & 6732 & 3.01 & 3.81 & 0.257 & 0.200 & 0.15 & 0.117 \\
\hline
\end{tabular}

The chromosome length, the percentage of SNP per chromosome, the heterozygous values $(\mathrm{H})$ and the polymorphism information content (PIC) are also reported

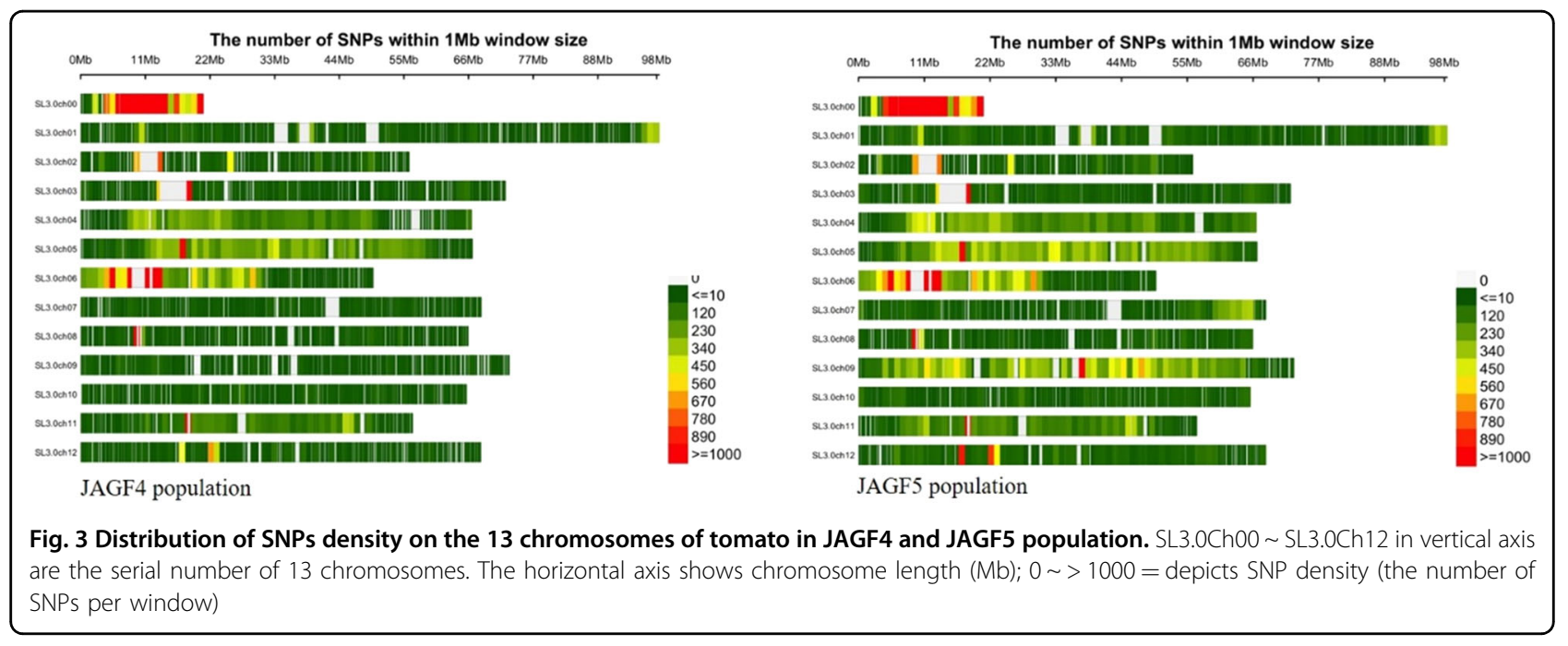

the correlation between GEBV and phenotype value in the TST, were 0.729 for YP and 0.715 for SSC, respectively. The correlation values between GEBVs and real phenotypic values for each of the 1000 iteration cycles were also displayed in Fig. 5.

GS model application in following generations to identify elite lines

The trained models build up in JAGF4 population were applied in next selection round to estimate GEBVs of F5 progeny population (JAGF5). After JAGF5 SNP dataset analysis, 10,648 common SNPs between the two populations were selected among high-quality SNPs and used for predicting GEBVs in JAGF5 and JAG8810 hybrid. In particular, the GEBVs of JAGF5 not phenotyped individuals were calculated by using the GS models trained on the JAGF4 individuals. Results obtained in this selection round are summarized in Table 3. To select the best and worst predicted performers, the JAG8810 GEBVs (7.9 and 4.6 for YP and SSC respectively) were used as threshold. Individuals with GEBVs higher than JAG8810 were predicted to be the best JAGF5 performers within the studied population. In the last step, the F6 offspring deriving from F5 plants and JAG8810 were phenotyped under heat stress condition to validate the goodness of prediction at phenotypic level for both analyzed traits (YP and SSC) and 


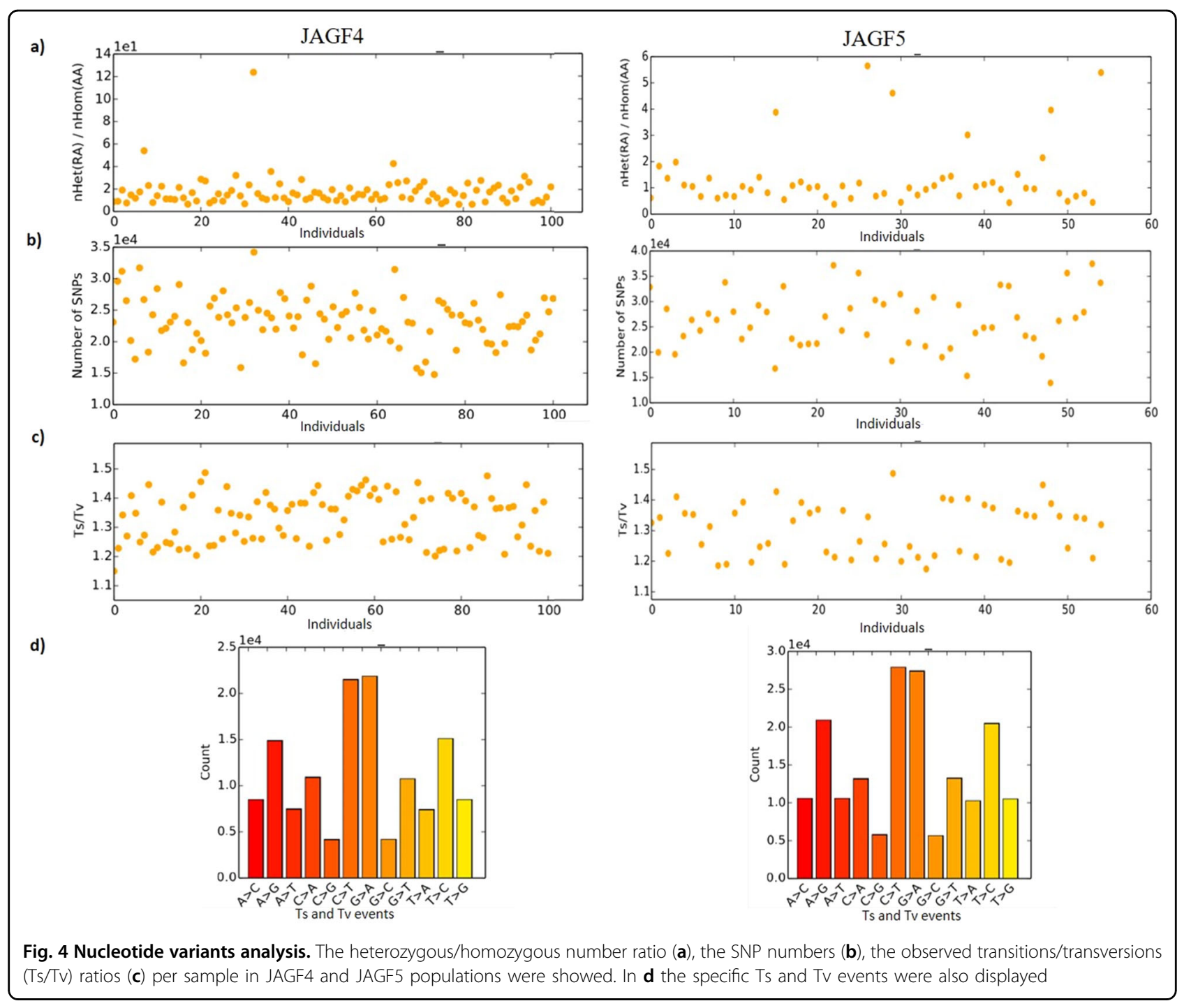

the best performers were selected among plants expressing phenotypic values higher than JAG8810 (Table 3).

Correlations between the GEBVs calculated on F5 genomic data and the phenotypic values obtained in F6 offspring were 0.67 and 0.68 for YP and SSC, respectively. Once the model accuracy was validated also in the next selection round, we identified 8 best performers for YP and 23 for SSC which were predicted among the JAGF5 population and confirmed in the phenotyped JAGF6 (Table 4).

Selected genotypes could be used as extremely promising elite lines in advanced breeding programs (Fig. 6).

\section{Variant effect prediction and QTL analysis to highligh loci involved in heat tolerance}

To identify novel alleles contributing to heat tolerance, we performed an integrative analysis on our SNP dataset.
A genomic variant annotation (defined as assignment of variant function) of all 10,648 high confidence SNPs was conducted. As showed in Fig. 7, the largest proportion of the SNPs was categorized as intergenic regions (78.1\%) whereas only $21.9 \%$ of SNPs were in gene body regions. In particular, variants located in UTRs, upstream and downstream regions accounted for $15.6 \%$. A small proportion of SNPs were categorized as intron variants (4\%), synonymous variants and missense variants (2\%). Instead, the category "other" included splice acceptor variant, splice donor variant, splice region variants, and stop gained which accounted for only $0.3 \%$. Within the derived SNPs subset of 2,278 SNPs in gene body regions, the variants with a modifier and low effect on the proteins were 2185 (accounted for 96\%). Those with a moderate effect were $89(3.9 \%)$ whereas 4 SNPs $(0.1 \%)$ showed a disruptive or high impact effect on the corresponding proteins. 

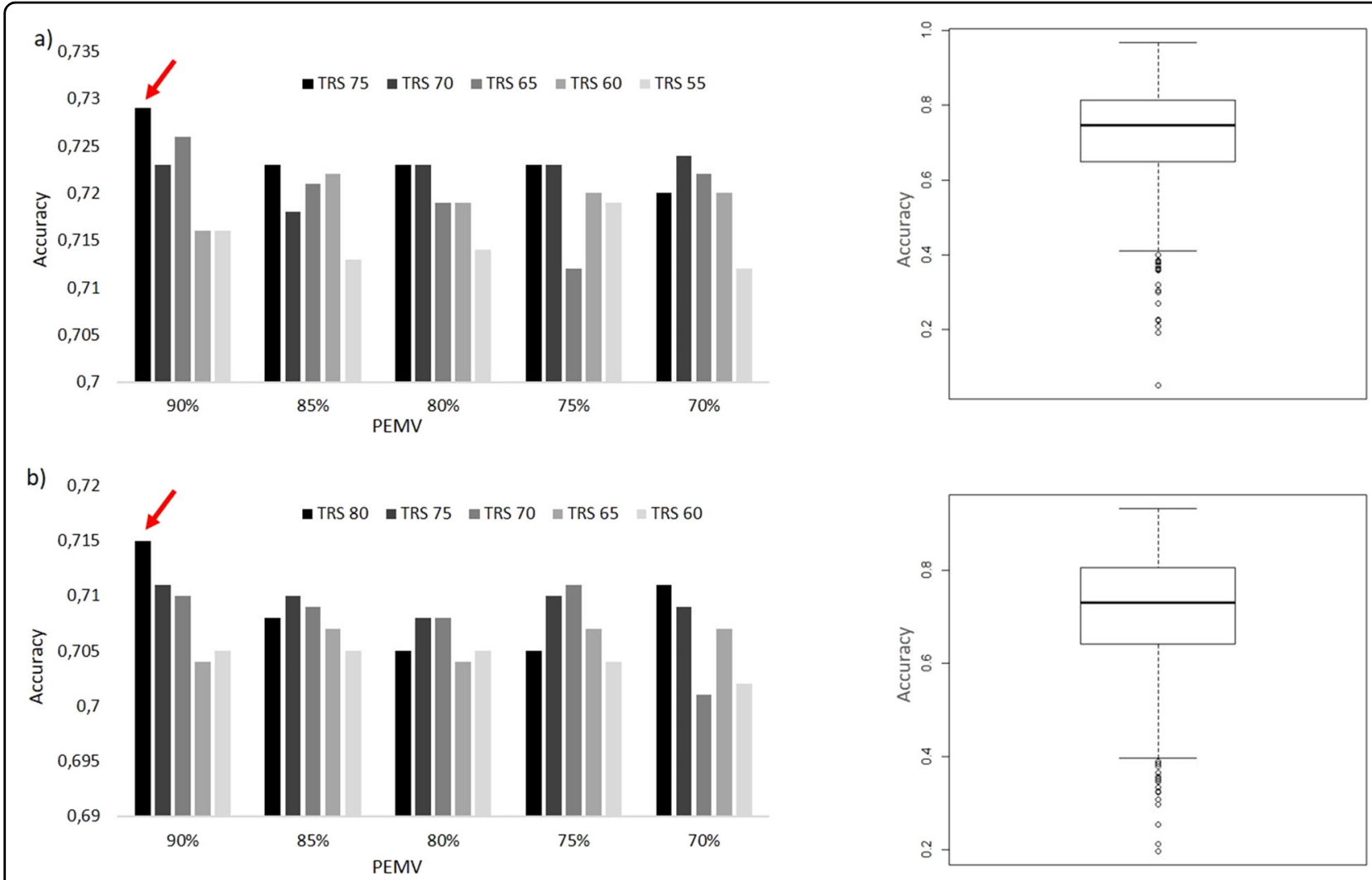

Fig. 5 On the left prediction accuracies under different proportion of TRS and PEMVs. The red arrow indicates the best combination for YP (a) and SSC (b). On the right, the plot between correlation values and 1000 cycles of iteration was displayed for the best combinations

Table 3 Summary of prediction and validation values in JAGF5 and JAGF6 populations

\begin{tabular}{lllll}
\hline & YP GEBVs JAGF5 & SSC GEBVs JAGF5 & YP values JAGF6 & SSC values JAGF6 \\
\hline JAG8810 & 7.9 & 4.6 & 3.5 & 4.3 \\
\% Best & 57.4 & 42.6 & 16.7 & 70.4 \\
\% Worst & 42.6 & 57.4 & 83.3 & 29.6 \\
\hline
\end{tabular}

GEBVs and phenotypic values of JAG8810 were reported for YP and SSC. \% of the best and worst values of predicted GEBVs in JAGF5 and phenotypic values in JAGF6 population were also reported

The 93 SNPs classified as having moderate and high impact were further analyzed in more detail. They targeted 52 genes for which the Solyc ID, the number of variants per gene, the predicted impact of the mutation, and the protein function are reported in Table 5 . The 52 genes affected by these variations are distributed on all chromosomes except chromosome 7 and 9 and some of them could be involved in abiotic stress responses.

To highlight putative important loci, a QTL analysis for YP based on JAGF4 population dataset was also performed (Supplementary Fig. 2). Three major QTLs on chromosome 3, 6, and 11 explaining 70, 86, and 53\% of the phenotypic variance respectively were identified. Interestingly, the QTL region on chromosome 3 underlined Solyc03g046596.1 and Solyc03g070435.1 variant genes with moderate impact, coding for BCL-2associated athanogene 6 and Alpha-mannosidase, respectively (Table 5). The large QTL chromosome region on chromosome 6 contained Solyc06g007530.2, Solyc06g008720.3, Solyc06g009920.1, Solyc06g036260.3, and Solyc06g036485.1 variant genes whereas on chromosome 11 QTL region Solyc11g028270.1, Solyc11g045677.1, and Solyc11g045260.1 variant genes, with moderate or high impact, coding for Bidirectional sugar transporter SWEET, vesicle-associated protein 1-4 and Photosystem II CP43 reaction center protein respectively, were found (Table 5). In addition, a QTL on chromosome 9 explaining the $51 \%$ of the phenotypic 
Table 4 Best JAGF5 predicted lines and JAGF6 best performers for YP and SSC were reported

\begin{tabular}{|c|c|c|c|c|}
\hline \multirow[b]{2}{*}{ Lines } & \multicolumn{2}{|l|}{ YP } & \multicolumn{2}{|l|}{ SSC } \\
\hline & Best JAGF5 & Best JAGF6 & Best JAGF5 & Best JAGF6 \\
\hline S2 & $\checkmark$ & $\checkmark$ & $x$ & $\checkmark$ \\
\hline S5 & $\checkmark$ & $x$ & $x$ & $\checkmark$ \\
\hline $\mathrm{S} 20$ & $x$ & $x$ & $\checkmark$ & $\checkmark$ \\
\hline S22 & $\checkmark$ & $\checkmark$ & $x$ & $\checkmark$ \\
\hline $\mathrm{S} 30$ & $\checkmark$ & $\checkmark$ & $x$ & $x$ \\
\hline S31 & $\checkmark$ & $x$ & $\checkmark$ & $\checkmark$ \\
\hline $\mathrm{S} 33$ & $x$ & $x$ & $\checkmark$ & $\checkmark$ \\
\hline S34 & $\checkmark$ & $x$ & $x$ & $\checkmark$ \\
\hline S36 & $x$ & $x$ & $\checkmark$ & $\checkmark$ \\
\hline S39 & $x$ & $x$ & $\checkmark$ & $\checkmark$ \\
\hline 541 & $\checkmark$ & $\checkmark$ & $\checkmark$ & $x$ \\
\hline 560 & $\checkmark$ & $x$ & $x$ & $\checkmark$ \\
\hline 562 & $x$ & $x$ & $x$ & $\checkmark$ \\
\hline 567 & $\checkmark$ & $x$ & $\checkmark$ & $\checkmark$ \\
\hline S68 & $x$ & $x$ & $x$ & $\checkmark$ \\
\hline S75 & $\checkmark$ & $x$ & $x$ & $x$ \\
\hline S76 & $x$ & $x$ & $\checkmark$ & $\checkmark$ \\
\hline S77_1 & $\checkmark$ & $x$ & $x$ & $x$ \\
\hline S77_2 & $\checkmark$ & $x$ & $x$ & $x$ \\
\hline S91 & $\checkmark$ & $x$ & $x$ & $x$ \\
\hline 592 & $\checkmark$ & $x$ & $x$ & $x$ \\
\hline S96 & $x$ & $x$ & $x$ & $x$ \\
\hline 597 & $x$ & $x$ & $\checkmark$ & $\checkmark$ \\
\hline S98 & $\checkmark$ & $x$ & $x$ & $x$ \\
\hline S99 & $x$ & $x$ & $\checkmark$ & $\checkmark$ \\
\hline S104 & $\checkmark$ & $x$ & $x$ & $x$ \\
\hline S105 & $\checkmark$ & $x$ & $x$ & $\checkmark$ \\
\hline 5112 & $x$ & $x$ & $\checkmark$ & $\checkmark$ \\
\hline S117 & $x$ & $x$ & $\checkmark$ & $\checkmark$ \\
\hline 5118 & $\checkmark$ & $x$ & $x$ & $x$ \\
\hline S121 & $x$ & $x$ & $\checkmark$ & $\checkmark$ \\
\hline S126 & $x$ & $x$ & $\checkmark$ & $\checkmark$ \\
\hline S127 & $x$ & $x$ & $\checkmark$ & $\checkmark$ \\
\hline 5128 & $x$ & $x$ & $\checkmark$ & $\checkmark$ \\
\hline S130 & $x$ & $x$ & $\checkmark$ & $\checkmark$ \\
\hline S132_1 & $\checkmark$ & $x$ & $x$ & $x$ \\
\hline S132_2 & $\checkmark$ & $x$ & $x$ & $\checkmark$ \\
\hline
\end{tabular}

Table 4 continued

\begin{tabular}{|c|c|c|c|c|}
\hline \multirow[b]{2}{*}{ Lines } & \multicolumn{2}{|l|}{ YP } & \multicolumn{2}{|l|}{ SSC } \\
\hline & Best JAGF5 & Best JAGF6 & Best JAGF5 & Best JAGF6 \\
\hline S139 & $x$ & $x$ & $\checkmark$ & $\checkmark$ \\
\hline S141 & $\checkmark$ & $x$ & $x$ & $x$ \\
\hline S151 & $x$ & $x$ & $\checkmark$ & $\checkmark$ \\
\hline S153 & $\checkmark$ & $\checkmark$ & $x$ & $\checkmark$ \\
\hline S159 & $\checkmark$ & $x$ & $x$ & $\checkmark$ \\
\hline S160 & $x$ & $x$ & $\checkmark$ & $\checkmark$ \\
\hline S179 & $x$ & $x$ & $\checkmark$ & $\checkmark$ \\
\hline S180 & $\checkmark$ & $x$ & $x$ & $\checkmark$ \\
\hline S185_1 & $\checkmark$ & $x$ & $x$ & $x$ \\
\hline S185_2 & $\checkmark$ & $x$ & $x$ & $\checkmark$ \\
\hline S194 & $\checkmark$ & $x$ & $x$ & $x$ \\
\hline S196 & $\checkmark$ & $\checkmark$ & $x$ & $x$ \\
\hline S205 & $\checkmark$ & $\checkmark$ & $x$ & $\checkmark$ \\
\hline S218 & $x$ & $x$ & $\checkmark$ & $\checkmark$ \\
\hline S225_1 & $\checkmark$ & $\checkmark$ & $x$ & $\checkmark$ \\
\hline S225_2 & $\checkmark$ & $x$ & $x$ & $\checkmark$ \\
\hline S239 & $x$ & $x$ & $\checkmark$ & $\checkmark$ \\
\hline
\end{tabular}

$\checkmark$ indicate lines with GEBV and/or phenotypic values higher than JAG8810; Xindicate lines with GEBV and/or phenotypic values lower than JAG8810

variance was identified. This QTL underlined 4 genes, Solyc09g042480.2, Solyc09g031970.3, Solyc09g031975.1, and Solyc09g031780.3, showing SNPs with a modifier impact and coding for chromatin remodeling complex subunit, glycogen/starch/alpha-glucan phosphorylase, $60 \mathrm{~S}$ acidic ribosomal protein family and chloroplast inner envelope family protein respectively.

Finally, we re-trained the GS models on the JAGF4 population retrieving only SNPs located in gene body regions and this resulted in a new optimized dataset of 2278 SNPs. To evaluate the prediction ability of the revised RR-blup models, 1000 cycles of iteration were run, using the optimized parameters previously described. Limiting the SNP dataset to a subset of 2278 SNPs in gene body regions did not significantly affected the accuracies of trained GS models if compared with previous models trained using the overall SNPs dataset ( 0.721 vs 0.729 for YP). By contrast a slight worsen accuracy (0.701) was observed using a dataset of 2278 SNPs randomly chosen from the starting dataset of 10,648 SNPs.

\section{Discussion}

Currently, tomato breeding is worldwide prospectively adapting conventional goals to the predicted scenario of 


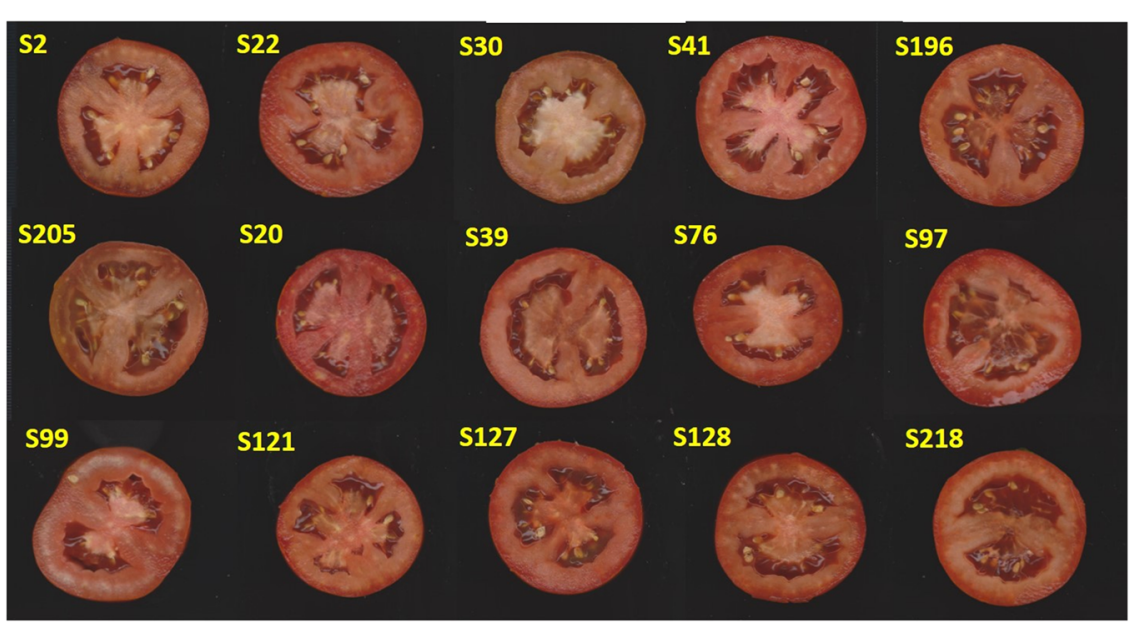

Fig. 6 Fruit longitudinal section of selected elite lines. Fruit longitudinal sections were shown

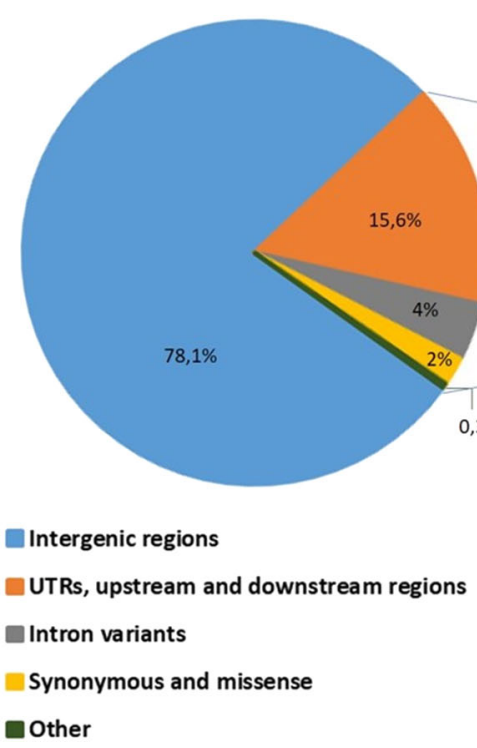

Fig. 7 Annotation of 10.648 high-confidence SNPs using SnpEff showing the classification of SNPs based on effects. The effect of SNP located in genic regions was also reported

changing environmental conditions as consequence of the global warming. In this view, the development of new high-yielding varieties tolerant to challenging hightemperature events appear to be of increasing interest. In this paper, we propose for the first time a GS approach based on a Single Seed Descendent (SSD) schema implemented in a population deriving from a heat tolerant tomato variety. The obtained F4 segregating progenies were screened for agronomic performance under heat stress conditions and genotyped to build up a GS model. Phenotypic evaluation revealed that traits related to fruit production such as FS, YP, and TFN, which are usually highly affected by high temperatures in tomatoes ${ }^{42,43}$, showed a wide variation. Yield positively correlated with other production-related traits such as TFN, FS, IN, FRL, confirming that they are primary traits involved in final yield performance under heat tolerance ${ }^{5,44-46}$. By contrast, yield-related traits resulted negatively correlated to SSC and CR indicating that increase in yield attributes results in decrease in quality components. Fruit ripening is a complex process involving numerous physiological and biochemical changes and uneven ripening probably reduced yield being out from sugar upsetting the source/ sink relationships. It is widely reported that tomato varieties with high SSC tend to be less productive ${ }^{47}$, although it represents an important tomato selection parameter. The number of diverse factors involved in yield under heat stress conditions clearly suggested that, overall, the 
Table 5 Solyc ID, number of variants, SNP impact and function of the 93 selected variants identified were reported

\begin{tabular}{|c|c|c|c|}
\hline Solyc ID & Number of SNP & SNP impact & Function \\
\hline Solyc00g008040.1 & 3 & Moderate & alpha/beta-Hydrolases superfamily protein \\
\hline Solyc00g012704.1 & 10 & Moderate & Pex2/Pex12 N-terminal domain-containing protein \\
\hline Solyc01g013910.1 & 1 & Moderate & Endoribonuclease Dicer 2b \\
\hline Solyc01g107700.3 & 1 & Moderate & Kynurenine formamidase \\
\hline Solyc01g108047.1 & 4 & Moderate & GATA transcription factor-like protein \\
\hline Solyc01g108083.1 & 7 & Moderate & Glucosamine--fructose-6-phosphateaminotransferase (Isomerizing) \\
\hline Solyc01g108320.3 & 3 & Moderate & Peroxidase \\
\hline Solyc01g108460.2 & 1 & Moderate & Serine carboxypeptidase 1 \\
\hline Solyc01g109150.4 & 2 & Moderate & cytochrome P450 CYP74C4 \\
\hline Solyc01g109160.4 & 1 & Moderate & cytochrome P450 CYP74C4 \\
\hline Solyc01g110820.3 & 1 & Moderate & RNA-binding protein 40; \\
\hline Solyc01g110995.1 & 1 & Moderate & 4-coumarate:CoA ligase 1 \\
\hline Solyc01g111240.3 & 3 & Moderate & Translocase of chloroplast 90, chloroplastic \\
\hline Solyc01g111430.2 & 2 & Moderate & Pentatricopeptide repeat-containing protein \\
\hline Solyc01g111620.3 & 1 & Moderate & AT4G35080-like protein (Fragment); Nickel/cobalt transporter, high-affinity \\
\hline Solyc01g111630.3 & 1 & Moderate & D-3-phosphoglycerate dehydrogenase \\
\hline Solyc01g111870.2 & 1 & Moderate & Serine/threonine-protein kinase \\
\hline Solyc01g111980.3 & 4 & Moderate & Lysine/histidine transporter \\
\hline Solyc01g112130.3 & 1 & Moderate & Dimethylaniline monooxygenase 5 \\
\hline Solyc01g112330.1 & 1 & Moderate & ARID/BRIGHT DNA-binding domain-containing protein \\
\hline Solyc02g085970.1 & 1 & Moderate & FACT complex subunit SSRP1 \\
\hline Solyc03g046596.1 & 1 & Moderate & BCL-2-associated athanogene 6 \\
\hline Solyc03g070435.1 & 1 & Moderate & Alpha-mannosidase \\
\hline Solyc03g113310.1 & 1 & Moderate & Pseudouridine synthase family protein \\
\hline Solyc03g113890.1 & 1 & Moderate & Zinc finger protein 6 \\
\hline Solyc03g114040.3 & 1 & Moderate & Os04g0347800 protein (Fragment) \\
\hline Solyc04g015130.3 & 2 & Moderate & Ribosomal protein S6 kinase alpha-3 \\
\hline Solyc04g074880.3 & 1 & Moderate & Purine permease family protein \\
\hline Solyc04g080610.3 & 1 & Moderate & Ornithine carbamoyltransferase \\
\hline Solyc05g025530.1 & 1 & Moderate & DNA-directed RNA polymerase subunit beta \\
\hline Solyc05g025540.1 & 1 & Moderate & Molybdenum cofactor sulfurase \\
\hline Solyc06g006057.1 & 2 & Moderate & Leucine-rich receptor-like protein kinase family protein \\
\hline Solyc06g007310.3 & 1 & Moderate & Deoxyribonuclease tatD \\
\hline Solyc06g007530.2 & 1 & Moderate & B3 domain-containing protein Os05g0481400 \\
\hline Solyc06g008720.3 & 1 & Moderate & Zinc ion binding protein \\
\hline Solyc06g009920.1 & 4 & Moderate & ATPase E1-E2 type family protein / haloacid dehalogenase-like hydrolase family protein \\
\hline Solyc06g036260.3 & 1 & Moderate & Beta-carotene hydroxylase 1 \\
\hline Solyc06g036485.1 & 2 & Moderate & Kinase family protein \\
\hline Solyc06g051190.2 & 1 & Moderate & RNA-dependent RNA polymerase family protein \\
\hline
\end{tabular}


Table 5 continued

\begin{tabular}{llll}
\hline Solyc ID & Number of SNP & SNP impact & Function \\
\hline Solyc08g062990.1 & 1 & Moderate & Protein kinase superfamily protein \\
Solyc10g061840.2 & 2 & Moderate & Carboxyl methyltransferase 4 \\
Solyc10g044430.1 & 2 & Moderate & FMN-linked oxidoreductases superfamily protein \\
Solyc11g028270.1 & 1 & Moderate & Bidirectional sugar transporter SWEET \\
Solyc11g039917.1 & 2 & Moderate & Retrovirus-related Pol polyprotein from transposon gypsy \\
Solyc11g039410.2 & 1 & Moderate & NAD(P)H-quinone oxidoreductase subunit K, chloroplastic \\
Solyc11g056540.1 & 4 & Moderate & Dynein-1-alpha heavy chain, flagellar inner arm I1 complex \\
Solyc11g061890.2 & 1 & Moderate & Adenylate isopentenyltransferase \\
Solyc12g076355.1 & 1 & Moderate & 3-oxo-5-alpha-steroid 4-dehydrogenase family protein \\
Solyc00g013184.1 & 1 & High & ubiquinone biosynthesis COQ9-like protein \\
Solyc05g026265.1 & 1 & High & Retrovirus-related Pol polyprotein from transposon TNT 1-94 \\
Solyc11g045677.1 & 1 & High & vesicle-associated protein 1-4 \\
Solyc11g045260.1 & 1 & High & Photosystem II CP43 reaction center protein \\
\hline
\end{tabular}

trait is under complex genetic control and addressing at the same time most of that factors would increase the chance to have success in term of selection gain.

Although GS has been previously applied for key productive traits such as YP and SSC in tomato ${ }^{31-33}$, there are no reports on GS implementation under heat stress conditions to our knowledge. Recent studies have demonstrated that the establishment of GS experiment optimal parameters such as TRN size, TRS and TST relatedness, marker density, precision of the phenotyping is relevant for a reliable prediction ${ }^{48-53}$. Training populations with strong relationship to the training set ensure higher prediction accuracies ${ }^{50}$. TRN design where individuals from the same family are used as both the TRN and TST has been extensively used in several breeding programs $^{54}$ such as in wheat ${ }^{55-57}$, maize ${ }^{58}$, rye ${ }^{59}$. Balancing LD present in initial segregating populations ${ }^{60,61}$ can help to obtain higher prediction accuracy also with a relatively small population size. Therefore, we trained our model in F4 segregating generation for both maintaining TRN-TST relationship and capturing long chromosome stretches in linkage disequilibrium (LD). We obtained a good prediction values ( 0.729 for YP and 0.715 for SSC) besides the limited population size (100 lines), highlighting the significance of full training-panel design. However, this type of design has some disadvantages since prediction models developed from single biparental populations have limited applications outside of particular selection scheme. Therefore, TRN designs that combine data from both related and unrelated families would be useful for plant breeders. In this case, the TRN must be created by using progenies of different genetic backgrounds, including full sibs, half-sibs, and other individuals with related ancestry. Of course, this TRN design has the great advantage of adapting well to implement GS in a wide range of breeding programs, but several studies have demonstrated that the prediction accuracy of GS substantially reduces especially when TRNs consisting of only unrelated individuals to the TST $^{62-65 .}$

At the same time our TRN was tested in combination with other parameters to improve the precision of model developed in JAGF4. In particular, genotyping-bysequencing (GBS) detected high-density markers with similar chromosome distribution as well as the heterozygous/homozygous ratio in both studied populations. The effects of Training Set size and GBS marker subsets filtering (by MAF and PEMV) on the model accuracy were evaluated in JAGF4 population to train the GS models. Starting from the complete dataset of 135,255 SNPs it was first filtered by MAF obtaining a dataset of 101,797 SNPs. Then our dataset was gradually reduced as PEMV increase, revealing that using a reduced subset of markers of $\sim 14,000$ filtered by PEMV of $90 \%$ did not impacted negatively on the overall prediction accuracy. This is an important point to take in account, since the use of fewer markers would result in significant cost reductions for genotyping but would also impact the extent of LD that is picked up by the prediction models. Moreover, our result clearly indicated that the marker dataset composed from the 10,648 high quality SNPs shared between the two generations (JAGF4 and JAGF5) was able to accurately track the genome of plants along the generations in order to successfully select individuals with the highest GEBV in JAGF5 and JAGF6. Our 
findings are in agreement with other studies that have shown that a reduced numbers of SNPs can be used in following stage of GS selection ${ }^{39-41,66,67}$. Indeed, our approach allowed the development of a GS model and its validation in field experimental trials reducing the number of generations and the size of the evaluated population. The GS models for YP and SSC obtained in this study efficiently predicted potential elite lines in F5 generation and accelerate selection for heat tolerant genotypes.

In addition, the GS performed on a subset of 2,278 variants mapped in gene body regions, suggests that the marker location is an important component of predictive ability of a genomic model since the accuracy was similar to the one obtained with the full dataset. Most of the 2,278 identified variants were located in UTRs, upstream and downstream regions that might highly affect transcription and/or translation and therefore might be useful for future applications $^{68}$. Moreover, the prediction of SNPs impact and the QTL analysis allowed the identification of four genes with high or moderate impact variants (Solyc03g046596, Solyc06g036260, Solyc11g045677, and Solyc11g028270), involved in thermotolerance and regulation of floral development and fruit set. The BAG (Bcl-2-associated-athanogene) Solyc03g046596 protein is a nucleotide exchange factors $^{69}$, involved both in programmed cell death ${ }^{70,71}$ and in basal thermotolerance ${ }^{72}$. BAG6 is also involved in the heat stress signal transduction pathway ${ }^{73,74}$ and in the re-folding of heat-denatured proteins ${ }^{75}$. The Beta-carotene hydroxylase Solyc06g036260 gene, involved in the xanthophyll cycle (the reversible interconversion of two carotenoids, violaxanthin, and zeaxanthin), has a key role in photoprotection ${ }^{76,77}$ and therefore it represents a promising target for genetic engineering to enhance heat stress tolerance ${ }^{78,79}$. Two interesting genes, located on chromosome 11, were also found. The Solyc11g045677, showing a SNP with high impact, encodes the vesicle-associated protein 1-4, important for maintaining homeostasis, cell growth and development, and polarity $^{80}$ during the regulation of abiotic stress response $\mathrm{e}^{81-83}$. The Solyc11g028270, with a moderate impact variant, encodes for Bidirectional sugar transporter SWEET, involved in the regulation of plant growth and development, a key traits for manipulating the carbohydrate partitioning process $^{84-86}$ to improve abiotic stress tolerance and yield. Moreover, on chromosome 9 QTL region is located the Solyc09g042480 gene, involved in chromatin remodeling complex (CHR) synthesis, that display a SNP with a modifier impact. It is widely reported that CHRs are implicated in the plant response to heat stress ${ }^{87,88}$. Therefore, genetic variability highlighted into the 5 tomato loci could account for altered control of critical processes leading to heat tolerance.

\section{Conclusions}

In the present study, different strategies were integrated to promote both the identification of traits involved in high temperature response and the selection of superior genotypes tolerant to heat stress in tomato. Optimized genomic prediction models for yield production and soluble solid content were developed, showing that GS is a valuable strategy to accelerate breeding for heat tolerance in tomato. In addition, the prediction of variant impact performed on our marker dataset integrated with the QTL analysis allowed us to select putative loci involved in high temperatures response to be further investigate. The GS model build up with the subset of SNPs located in gene body regions showed an accuracy similar to the one obtained with the full dataset, suggesting that the marker location is an important component of predictive ability of our model.

\section{Materials and methods}

\section{Field trials and phenotypic evaluation of segregating populations}

The tomato variety JAG8810, kindly obtained from Bayer station (Latina, Italy) was self-pollinated from F2 to F4 generations through a Single Seed Descendent (SSD) schema (Supplementary Fig. 3) in greenhouse of the Department of Agriculture of the University of Naples Federico II (N 40 48.' 49.352"'; E $14^{\circ} 20^{\prime} 40.073^{\prime \prime}$ ). In 2017, 100 F4 lines (JAGF4) were grown in open field in Southern Italy region (Battipaglia-Campania; N $40^{\circ} 58 .^{\prime}$ $56.69^{\prime \prime}$; E $\left.14^{\circ} 96^{\prime} 10.02^{\prime \prime}\right)$, usually characterized by high temperatures during the flowering and fruit set periods (from June to August). Five seedlings for each JAGF4 line were transplanted in field under plastic tunnel in a completely randomized design in the beginning of May 2017 in order to expose plants to high temperatures. Tomato plants were grown following the standard cultural practices of the area and temperature and climatic data were recorded using the weather station VantagePro2 (Davis Instrument Corporation).

Three random plants per genotype were analyzed for traits related to flowering, fruit production and quality. For four of these traits (fruit earliness (FRL), leaf coverage (LC), inflorescences number (IN) and contemporaneous ripening (CR) a score (from one to five) was calculated. The percentage of fruit set (FS) were evaluated on inflorescences produced from the second to the fifth truss on three plants per line randomly chosen. Finally, at fruit red ripe stage, total fruit number (TFN), soluble solid content (SSC-3 fruits per plant) and yield production per plant (YP) were measured. Correlation analyses was carried out using R package "corrplot" ${ }^{89}$. Fifty-four JAGF6 offspring plants deriving from the JAGF5 population and the hybrid JAG8810 were grown and phenotyped in 2019 as described for JAGF4 population (Battipaglia-Campania; $\mathrm{N} 40^{\circ} 58 .^{\prime} 56.69^{\prime \prime}$; E $14^{\circ} 96^{\prime} 10.02^{\prime \prime}$ ) except for the planting in double rows. At fruit red ripe stage, the Soluble Solid 
Content (SSC-3 fruits per plant) and yield production per plant (YP) were measured in order to validate the model.

\section{DNA extraction and sequencing}

DNA was extracted from $100 \mathrm{mg}$ of young leaf tissues from JAG8810, 100 JAGF4, and 54 JAGF5 single individuals, using the Qiagen DNeasy Plant kit (Qiagen, Hilden, Germany). The DNA concentration was estimated using the Qubit fluorometer (Invitrogen, Carlsbad, CA, USA) and the 260/280 and 260/230 ratios were assayed using the NanoDrop 1000 spectrophotometer (Thermo Fischer, Waltham, MA, USA). DNA samples were sent to the IGA technology services (Udine, IT) for genotyping by sequencing (GBS) approach. In silico analysis on the reference genome was used to select the best combination of the two restriction enzymes Sph $I$ and $M b o I$ and the best fragment size distribution to obtain the desired number of loci. Libraries were processed with Illumina cBot for cluster generation on the flowcell, following the manufacturer's instructions and sequenced with V4 chemistry paired end $125 \mathrm{bp}$ mode on HiSeq2500 instrument (Illumina, San Diego, CA).

\section{Read mapping, variant calling, and annotation}

A quality check was performed on the raw sequencing data to remove low quality portions while preserving the longest high quality part of NGS reads. The minimum length was set to $35 \mathrm{bp}$ and the quality score to 25 . The software BBDuk was used for this scope.

The high-quality reads were aligned against the Solanum lycopersicum reference genome sequence (SL3.0) with BWA aligner ${ }^{90}$. Default parameters and selection of uniquely aligned reads (i.e., reads with a mapping quality $>4$ ) were used. Filtering of detected loci was carried out using the Populations program included in Stacks v2.0 ${ }^{91}$. Populations was run with option $-r=0.75$ in order to retain only loci that are represented in at least the $75 \%$ of the population. Within the Stacks v2.0 package, the "ref_map.pl" program was used for variant calling ${ }^{90}$. This program aligned data to the reference genome. A VCF file was created and filtered by Minimum Read Depth (DP) > $=4$. Variant Calling Filter Files (VCFs) containing all the identified variants were obtained. The VCF file were subjected to a filtering procedure using Vcftools v.0.1.13 (http://vcftools.sourceforge.net/) setting the percentage of eliminated missing values at different levels and the minor allele frequency at 0.05 .

SNP data in VCF format are publicly available at FIGSHARE with the following link (https://figshare.com/s/ 9c64512c04c5bc220e22). Users can download and use the data for research purpose with acknowledgment to authors. In order to perform a variant annotation of the Variant Calling Files (VCF), the SnpEff ${ }^{92}$ was employed on JAGF4 population dataset, which let the association of each variant to the annotated genes and to predict their effect on the protein function. Variants were classified based on their location (e.g., exon, intron, intergenic regions, splice sites, etc.) and the effect of their 'impact': High, Moderate, Low and Modifier.

\section{Genomic selection model construction and optimization}

The 100 phenotyped and genotyped JAGF4 individuals were used to build up a TRN. The TRN was divided in TRS e TST in a hold-out validation scheme, meaning that the model was build using $X$ individuals of the TRS and validated in the TST with remaining 100-X lines. Genomic breeding values (GEBVs) for YP and SSC were estimated using ridge regression best linear unbiased prediction $^{93}$.

The predictive ability of genomic selection models was evaluated calculating the Pearson correlation between the phenotypic values and the GEBVs (predicted) values across 1000 cycles of iteration.

The hold-out validation was implemented with five different samples sizes in test set $(15,20,25,30$, and 35 for YP and 20, 25, 30, 35, 40 for SSC). For each sample size, five different set of SNPs filtered by minor-allele frequency $(\mathrm{MAF})>0.05$, based on different levels of percentage of eliminated missing values (PEMVs: 90, 85, 80, $75,70 \%$ ), were used. After filtering, remaining missing values were imputed using the EM method with the $A$. mat function of the "rrBLUP" package in $\mathrm{R}^{93}$. Therefore, a total 25 models with different sample sizes and number of SNPs were compared. For each of the 25 models, random sampling was repeated 1000 times. The best combination was selected to calculate the final accuracies of the trained models. After JAGF5 SNP dataset analysis, 10,648 common SNPs between the two populations were selected among high-quality SNPs (Table S5). This SNP dataset and the phenotypic values of the $100 \mathrm{~F} 4$ individuals were used as TRS to estimate GEBVs of F5 progeny population. A phenotypic characterization under heat stress conditions in F6 offspring deriving from F5 population was employed to validate the model.

\section{SNP dataset evaluation}

Starting from the previously SNP dataset used for the JAGF5 prediction model, SNP located in intergenic region were removed and the predictive ability of genomic selection models was evaluated calculating the Pearson correlation between the phenotypic values and the GEBVs (predicted) across 1000 cycles of iteration in the original training population (F4).

\section{Quantitative trait locus (QTL) analysis}

QTL analyses for YP was performed on JAGF4 population using R-based software package, $\mathrm{R} / \mathrm{qtl}^{94,95}$ in $\mathrm{R}$ version 4.0.5. The dataset of 10,648 common SNPs 
between JAGF4 and JAGF5 individuals was used. Frist SNP markers were coded as $\mathrm{AA}, \mathrm{BB}, \mathrm{AB}$, and $\mathrm{NA}$ respectively for major allele, minor allele, heterozygosity and missing data. Then, genetic maps were constructed by est.map using the Kosambi's function of R-qtl for the conversion of recombination frequency to genetic distance $^{96}$. Composite interval mapping (CIM) was applied for QTL detection using the maximum likelihood via the expectation-maximization algorithm ${ }^{97}$ with a window size (window) of $15 \mathrm{cM}$ and the marker covariate (n.marcovar) equals to 4 . Finally, significance thresholds were generated for each trait by using the permutation test $(\alpha=0.05, n=$ 1000). The percentage phenotypic variance explained by a QTL was estimated via the following formula: $h 2=1-10$ $\left(-2^{*} \mathrm{LOD} / \mathrm{n}\right)$, where "n" is the sample size and "LOD" the single-QTL model LOD score, using single interval mapping (SIM). The whole genome was scanned in steps of $1 \mathrm{cM}$ by the scanone function. The $95 \%$ Bayes credible interval was assessed using the bayesint function, using the LOD score of CIM.

\section{Acknowledgements}

The authors have received funding from the European Union's Horizon 2020 research and innovation programme through the TomGEM project under grant agreement No 679796 and through the HARNESSTOM project under grant agreement No 101000716.

\section{Author contributions}

E.C. wrote the manuscript and was centrally involved in analysis and interpretation of data. G.A. was involved in field trials and revised the manuscript. A.G. performed QTL analysis and interpretation. A.D.M. supervised the statistical analysis and substantially revised the text. A.B. provided important suggestions for improving the manuscript. L.F. supervised field trials and critically revised the manuscript. M.R.E. conceived the study, coordinated work, and contributed to data interpretation and manuscript writing. All the authors read and approved the final manuscript.

\section{Data availability}

Data obtained are publicly available at https://doi.org/10.6084/m9. figshare.14937795.

\section{Conflict of interest}

The authors declare no competing interests.

Supplementary information The online version contains supplementary material available at https://doi.org/10.1038/s41438-021-00647-3.

Received: 27 February 2021 Revised: 5 July 2021 Accepted: 14 July 2021 Published online: 01 October 2021

\section{References}

1. Frusciante, L. et al. Evaluation and use of plant biodiversity for food and pharmaceuticals. Fitoterapia 1, 66-72 (2000).

2. Sato, S., Peet, M. M. \& Thomas, J. F. Determining critical pre- and post-anthesis periods and physiological processes in Lycopersicon esculentum Mill. exposed to moderately elevated temperatures. J. Exp. Bot. 53, 1187-1195 (2002).

3. Gerszberg, A. \& Hnatuszko-Konka, K. Tomato tolerance to abiotic stress: a review of most often engineered target sequences. Plant Growth Regul. 83, 175-198 (2017).

4. Bailey-Serres, J., Lee, S. C. \& Brinton, E. Waterproofing crops: effective flooding survival strategies. Plant Physiol. 160, 1698-1709 (2012).
5. Ayenan, M. A. T. et al. Accelerating breeding for heat tolerance in tomato (Solanum lycopersicum L.): an integrated approach. Agronomy 9, 720 (2019).

6. Luo, Q. Temperature thresholds and crop production: a review. Clim. Change 109, 583-598 (2011).

7. Wahid, A. Physiological implications of metabolite biosynthesis for net assimilation and heat-stress tolerance of sugarcane (Saccharum officinarum) sprouts. J. Plant Res. 120, 219-228 (2007).

8. Tayade, R., Nguyen, T., Oh, S. A. \& Hwang, Y. S. Effective strategies for enhancing tolerance to high-temperature stress in rice during the reproductive and ripening stages. Plant Breed. Biotechnol. 6, 1-18 (2018).

9. Rahim, M. S. et al. In Accelerated Plant Breeding: Cereal Crops (eds Gosal, S. S. \& Wani, S. H.) (Springer, 2020).

10. D'Esposito et al. Unraveling the complexity of transcriptomic, metabolomic and quality environmental response of tomato fruit. BMC Plant Biol. 17, 66 (2017).

11. Esposito, S. et al. ddRAD sequencing-based genotyping for population structure analysis in cultivated tomato provides new insights into the genomic diversity of Mediterranean 'da serbo' type long shelf-life germplasm. Hortic. Res 7, 134 (2020).

12. Esposito, S., Carputo, D., Cardi, T. \& Tripodi, P. Applications and trends of machine learning in genomics and phenomics for next-generation breeding. Plants 9, 34 (2020).

13. Guijarro-Real, C. et al. Large scale phenotyping and molecular analysis in a germplasm collection of rocket salad (Eruca vesicaria) reveal a differentiation of the gene pool by geographical origin. Euphytica 216, 53 (2020).

14. Cappetta, E. et al. Accelerating tomato breeding by exploiting genomic selection approaches. Plants 9, 1-14 (2020).

15. Cappetta, E., Andolfo, G., Di Matteo, A. \& Ercolano, M. R. Empowering crop resilience to environmental multiple stress through the modulation of key response components. J. Plant Physiol. 246-247, 153134 (2020).

16. $\mathrm{Xu}$, J. et al. Mapping quantitative trait loci for heat tolerance of reproductive traits in tomato (Solanum lycopersicum). Mol. Breed. 37, 58 (2017).

17. Zhang, S. et al. Detection of major loci associated with the variation of 18 important agronomic traits between Solanum pimpinellifolium and cultivated tomatoes. Plant J. 95, 312-323 (2018).

18. Driedonks, N. et al. Exploring the natural variation for reproductive thermotolerance in wild tomato species. Euphytica 214, 67 (2018).

19. Ruggieri, V. et al. Exploiting genetic and genomic resources to enhance heattolerance in tomatoes. Agronomy 9, 22 (2019).

20. Olivieri, F. et al. High-throughput genotyping of resilient tomato landraces to detect candidate genes involved in the response to high temperatures. Genes $11,626(2020)$

21. Jannink, J. L., Lorenz, A. J. \& Iwata, H. Genomic selection in plant breeding: from theory to practice. Brief. Funct. Genomics 9, 166-177 (2010).

22. Zhao, Y., Mette, M. F., Gowda, M., Longin, C. F. H. \& Reif, J. C. Bridging the gap between marker-assisted and genomic selection of heading time and plant height in hybrid wheat. Heredity 112, 638-645 (2014).

23. Bernardo, R. \& Yu, Y. Prospects for genome-wide selection for quantitative traits in maize. Crop Sci. 47, 1082-1090 (2007).

24. Heffner, E. L., Sorrells, M. E. \& Jannink, J. Genomic selection for crop improvement. Crop Sci. 49, 1-12 (2009).

25. Crossa, J. et al. Prediction of genetic values of quantitative traits in plant breeding using pedigree and molecular markers. Genetics 186, 713-724 (2010).

26. Lorenz, A. J. et al. Genomic selection in plant breeding. knowledge and prospects. Adv. Agron. 110, 77-123 (2011).

27. Contaldi, F., Cappetta, E. \& Esposito, S. In Crop Breeding Methods and Protocols (Springer, 2021).

28. Crossa, J. et al. Genomic prediction in CIMMYT maize and wheat breeding programs. Heredity 112, 48-60 (2013).

29. Song, J. et al. Practical application of genomic selection in a doubled-haploid winter wheat breeding program. Mol. Breed. 37, 117 (2017).

30. Cui, Y. et al. Hybrid breeding of rice via genomic selection. Plant Biotechnol. J. 18, 57-67 (2020)

31. Duangjit, J., Causse, M. \& Sauvage, C. Efficiency of genomic selection for tomato fruit quality. Mol. Breed. 36, 29 (2016).

32. Yamamoto, E. et al. A simulation-based breeding design that uses wholegenome prediction in tomato. Sci. Rep. 6, 19454 (2016).

33. Yamamoto, E. et al. Efficiency of genomic selection for breeding population design and phenotype prediction in tomato. Heredity $\mathbf{1 1 8}$ 202-209 (2017). 
34. Liabeuf, D., Sim, S. C. \& Francis, D. M. Comparison of marker-based genomic estimated breeding values and phenotypic evaluation for selection of bacterial spot resistance in tomato. Phytopathology 108, 392-401 (2018).

35. Crossa, J. et al. Genomic selection in plant breeding: methods, models, and perspectives. Trends Plant Sci. 22, 961-975 (2017).

36. Tan, B. et al. Evaluating the accuracy of genomic prediction of growth and wood traits in two Eucalyptus species and their F1 hybrids. BMC Genomics 17, 110 (2017).

37. Zhang, Z. et al. Improving the accuracy of whole genome prediction for complex traits using the results of genome wide association studies. PLOS ONE 9. e93017 (2014).

38. Su, G. et al. Comparison of genomic predictions using medium-density (similar to 54,000 ) and high-density (similar to 777,000 ) single nucleotide polymorphism marker panels in Nordic Holstein and Red Dairy Cattle populations J. Dairy Sci. 95, 4657-4665 (2012)

39. Gutierrez, A. P., Matika, O., Bean, T. P. \& Houston, R. D. Genomic selection for growth traits in Pacific oyster. Front Genet. 9, 391 (2018).

40. Palaiokostas, C. et al. Optimizing genomic prediction of host resistance to koi herpesvirus disease in carp. Front Genet. 10, 543 (2019).

41. Tsairidou, S., Anacleto, O., Woolliams, J. A. \& Doeschl-Wilson, A. Enhancing genetic disease control by selecting for lower host infectivity and susceptibility. Heredity 122, 742-758 (2019).

42. Sato, S. Moderate increase of mean daily temperature adversely affects fruit set of Lycopersicon esculentum by disrupting specific physiological processes in male reproductive development. Ann. Bot. 97, 731-738 (2006).

43. Golam, F., Prodhan, Z. H., Nezhadahmadi, A. \& Rahman, M. Heat tolerance in tomato. Life Sci. J. 9, 1936-1950 (2012).

44. Haydar, A. et al. Studies on genetic variability and unterrelationship among the different traits in tomato (Lycopersicon esculentum Mill.) Middle-East. J. Sci. Res. 2, 139-142 (2007)

45. Bernousi, I., Emami, A., Tajbakhsh, M., Darvishzadeh, R. \& Henareh, M. Studies on genetic variability and correlation among the different traits in Solanum Lycopersicum L. Notulae Botanicae Horti Agrobotanici Cluj-Napoca 39, 152-158 (2011).

46. Tasisa, J., Belew, D. \& Bantte, K. Genetic associations analysis among some traits of tomato (Lycopersicon esculentum Mill.) genotypes in West Showa, Ethiopia. Int J. Plant Breed. Genet. 6, 129-139 (2012).

47. Tigist, M., Workneh, T. S. \& Woldetsadik, K. Effects of variety on the quality of tomato stored under ambient conditions. J. Food Sci. Technol. 50, 477-486 (2013).

48. Robertsen, C. D., Hjotrtshøj, R. L. \& Janss, L. L. Genomic selection in cereal breeding. Agron 9, 1-16 (2019).

49. Cericola, F. et al. Optimizing training population size and genotyping strategy for genomic prediction using association study results and pedigree information. A case of study in advanced wheat breeding lines. PLOS ONE 12, e0169606 (2017).

50. Edwards, S. M. et al. The effects of training population design on genomic prediction accuracy in wheat. Theor. Appl Genet. 132, 1943-1952 (2019).

51. Jannink, J.-L., Lorenz, A. J. \& Iwata, H. Genomic selection in plant breeding: from theory to practice. Brief. Funct. Genomics 9, 166-177 (2010).

52. Hickey, L. T. et al. Breeding crops to feed 10 billion. Nat. Biotechnol. 37 744-754 (2019).

53. Moeinizade, S., Hu, G., Wang, L. \& Schnable, P. S. Optimizing selection and mating in genomic selection with a look-ahead approach: an operations research framework. G3: Genes Genom. Genet. 9, 2123-2133 (2019).

54. Combs, E. \& Bernardo, R. Accuracy of genome wide selection for different traits with constant population size, heritability, and number of markers. Plant Genome https://doi.org/10.3835/plantgenome2012.11.0030 (2013).

55. Herter, C. P., Ebmeyer, E., Kollers, S., Korzun, V. \& Miedaner, T. An experimental approach for estimating the genomic selection advantage for Fusarium head blight and Septoria tritici blotch in winter wheat. Theor. Appl. Genet. 132 2425-2437 (2019).

56. Ornella, L. et al. Genomic prediction of genetic values for resistance to wheat rusts. Plant Genome 5, 136-148 (2012).

57. Thavamanikumar, S., Dolferus, R. \& Thumma, B. R. Comparison of genomic selection models to predict flowering time and spike grain number in two hexaploid wheat doubled haploid populations. G3-Genes Genom. Genet. 5, 1991-1998 (2015).

58. Guo, T. et al. Performance prediction of F1 hybrids between recombinant inbred lines derived from two elite maize inbred lines. Theor. Appl. Genet. 126 189-201 (2013)
59. Wang, Y. et al. The accuracy of prediction of genomic selection in elite hybrid rye populations surpasses the accuracy of marker-assisted selection and is equally augmented by multiple field evaluation locations and test years. BMC Genomics 15, 556 (2014).

60. Lorenzana, R. E. \& Bernardo, R. Accuracy of genotypic value predictions for marker- based selection in biparental plant populations. Theor. Appl. Genet. 120, 151-161 (2009).

61. Zhao, Y. et al. Accuracy of genomic selection in European maize elite breeding populations. Theor. Appl. Genet. 124, 769-776 (2012).

62. Gowda, M. et al. Relatedness severely impacts accuracy of markerassisted selection for disease resistance in hybrid wheat. Heredity $\mathbf{1 1 2}$, 552-561 (2014).

63. Lehermeier, C. et al. Usefulness of multiparental populations of maize (Zea mays L.) for genome-based prediction. Genetics 198, 3-16 (2014).

64. Zhao, Y., Zeng, J., Fernando, R. \& Jochen Reif, C. Genomic prediction of hybrid wheat performance. Crop Sci. 53, 802-810 (2013).

65. Albrecht, T. et al. Genome-based prediction of testcross values in maize. Theor. Appl. Genet. 123, 339-350 (2011).

66. Zhang, Z., Ding, X., Liu, J., Zhang, Q. \& Koning, D. J. Accuracy of genomic prediction using low-density marker panels. J. Dairy Sci. 94, 3642-3650 (2011).

67. Correa, K., Bangera, R., Figueroa, R., Lhorente, J. P. \& Yáñez, J. M. The use of genomic information increases the accuracy of breeding value predictions for sea louse (Caligus rogercresseyi) resistance in Atlantic salmon (Salmo salar). Genet Sel. Evol. 49, 15 (2017).

68. Guajardo, V. et al. Genome-wide SNP identification in Prunus rootstocks germplasm collections using Genotyping-by-Sequencing: phylogenetic analysis, distribution of SNPs and prediction of their effect on gene function. Sci. Rep. 10, 1 (2020).

69. Doukhanina, E. V. et al. Identification and functional characterization of the BAG protein family in Arabidopsis thaliana. J. Biol. Chem. 281, 18793-18801 (2006).

70. Kang, C. H. et al. AtBAG6, a novel calmodulin-binding protein, induces programmed cell death in yeast and plants. Cell Death Differ. 13, 84-95 (2006).

71. Li, Y., Kabbage, M., Liu, W. \& Dickman, M. B. Aspartyl protease-mediated cleavage of BAG6 is necessary for autophagy and fungal resistance in plants. Plant Cell 28, 233-247 (2017).

72. Echevarría-Zomeño, S. et al. Dissecting the proteome dynamics of the early heat stress response leading to plant survival or death in Arabidopsis. Plant Cell Environ. 39, 1264-1278 (2016).

73. Wu, H. C., Luo, D. L., Vignols, F. \& Jinn, T. L. Heat shock-induced biphasic Ca(2+) signature and OsCaM1-1 nuclear localization mediate downstream signalling in acquisition of thermotolerance in rice (Oryza sativa L.). Plant Cell Environ. 35, 1543-1557 (2012)

74. Kityk, R., Kopp, J., Sinning, I. \& Mayer, M. P. Structure and dynamics of the ATP-bound open conformation of Hsp70 chaperones. Mol. Cell 48, 863-874 (2012).

75. Mayer, M. P. Hsp70 chaperone dynamics and molecular mechanism. Trends Biochem Sci. 38, 507-514 (2013).

76. Havaux, M. Carotenoid oxidation products as stress signals in plants. Plant J. 79, 597-606 (2014).

77. Jahns, P. \& Holzwarth, A. R. The role of the xanthophyll cycle and of lutein in photoprotection of photosystem II. Biochimica et. Biophysica Acta (BBA)-Bioenerg. 1817, 182-193 (2012).

78. Davison, P. A., Hunter, C. N. \& Horton, P. Overexpression of $\beta$-carotene hydroxylase enhances stress tolerance in Arabidopsis. Nature 418, 203-206 (2002).

79. He, J. et al. Heterologous expression of HpBHY and CrBKT increases heat tolerance in Physcomitrella patens. Plant Diversity 41, 266-274 (2019).

80. Blatt, M. R. \& Thiel, G. In The Golgi Apparatus and the Plant Secretory Pathway (Robinson, D. G., ed), 208-237 (Blackwell Publishing/CRC Press, 2003).

81. Leshem, Y., Golani, Y., Kaye, Y. \& Levine, A. Reduced expression of the v-SNAREs AtVAMP71/AtVAMP7C gene family in Arabidopsis reduces drought tolerance by suppression of abscisic acid-dependent stomatal closure. J. Exp. Bot. 61, 2615-2622 (2010)

82. Xue, Y., Yang, Y., Yang, Z., Wang, X. \& Guo, Y. VAMP711 is required for abscisic acid-mediated inhibition of plasma membrane $\mathrm{H}+$-ATPase activity. Plant Physiol. 178, 1332-1343 (2018).

83. Singh, B., Khurana, P., Khurana, J. P. \& Singh, P. Gene encoding vesicleassociated membrane protein-associated protein from Triticum aestivum (TaVAP) confers tolerance to drought stress. Cell Stress Chaperones 23, 411-428 (2018). 
84. Klemens, P. A. et al. Overexpression of the vacuolar sugar carrier AtSWEET16 modifies germination, growth, and stress tolerance in Arabidopsis. Plant Physiol. 163, 1338-1352 (2013).

85. Wei, Y., Xiao, D., Zhang, C. \& Hou, X. The expanded SWEET gene family following whole genome triplication in Brassica rapa. Genes 10, 722 (2019).

86. Julius, B. T., Leach, K. A., Tran, T. M., Mertz, R. A. \& Braun, D. M. Sugar transporters in plants: new insights and discoveries. Plant Cell Physiol. 58, 1442-1460 (2017).

87. Liu, J., Feng, L., Li, J. \& He, Z. Genetic and epigenetic control of plant heat responses. Front. Plant Sci. 6, 267 (2015).

88. Mlynarova, L., Nap, J. P. \& Bisseling, T. The SWI/SNF chromatin-remodeling gene AtCHR12 mediates temporary growth arrest in Arabidopsis thaliana upon perceiving environmental stress. Plant J. 51, 874-885 (2007).

89. Wei, T. \& Simko, V. R package "corrplot": Visualization of a Correlation Matrix (Version 0.84) (2017).

90. Li, H. \& Durbin, R. Fast and accurate short read alignment with BurrowsWheeler transform. Bioinformatics 25, 1754-1760 (2009).
91. Catchen, J., Hohenlohe, P. A., Bassham, S., Amores, A. \& Cresko, W. A. Stacks: an analysis tool set for population genomics. Introduces Stacks, a widely used software package for locus discovery, genotyping and population genomic analysis using RADseq data. Mol. Ecol. 22, 3124-3140 (2013).

92. Cingolani, P. et al. A program for annotating and predicting the effects of single nucleotide polymorphisms, SnpEff: SNPs in the genome of Drosophila melanogaster strain W1118; iso-2; iso-3. Fly 6, 80-92 (2012).

93. Endelman, J. B. New algorithm improves fine structure of the barley consensus SNP map. BMC Genomics 12, 407 (2011).

94. Broman, K. W., Wu, H., Sen, S. \& Churchill, G. A. R / qtl: QTL mapping in sperimental crosses. Bioinformatics 19, 889-890 (2003).

95. Broman, K. W. \& Sen, S. A. Guide to QTL Mapping with R/qtl. Statistic for Biology and Health. (2009)

96. Kosambi, D. D. The estimation of map distances from recombination values Ann. Eugen. 12, 172-175 (1944).

97. Dempster, A. P., Laird, N. M. \& Rubin, D. B. Maximum likelihood from incomplete data via the EM algorithm. J. R. Stat. Soc.: Ser. B (Methodol.) 39, $1-22$ (1977). 